\title{
LA INVESTIGACIÓN: MAS ALLÁ DEL RANKING DE LAS UNIVERSIDADES
}

\author{
RESEARCH: BEYOND THE RANKING OF THE UNIVERSITIES
}

Jhony A. De La Cruz -Vargas ${ }^{1, a, b, c, c}$, Elio Rodríguez-Chávez ${ }^{2, d, e, f}$

\begin{abstract}
Uno de los procesos más importantes y del cual se sostienen todas las demás actividades universitarias es la investigación. La investigación es creación, producción original, desarrollo de la argumentación y la coherencia de las ideas, revisión exhaustiva-reflexión profunda, resolver los misterios y, en última instancia, darle foco a los sueños. El enfoque conceptual integral, planteado por Rico', para quien la investigación "es colonizar una parcela pequeña o grande, sembrar, cultivar, cosechar y distribuir sus frutos, para que luego vengan otros a mejorar, optimizar y superar nuestra faena".
\end{abstract}

De manera metafórica con la siembra el proceso de la investigación incluye tres momentos: Un primer momento de sembrar y cultivar como la aplicación de la aproximación metódologica, un segundo momento de cosechar como la asimilación, interpretación y discusión de los resultados o hallazgos y, por último la distribución de sus frutos se puede entender como la difusión del conocimiento, sinónimo inequívoco de la publicación. A la universidad, como cuna de investigación, le corresponde la ardua tarea de integrar, apropiar y producir conocimientos, además de transmitirlos.

\section{Las 20 mejores Universidades en el Perú según SUNEDU y América Economía}

A principios de 2018, la Superintendencia Nacional de Educación Universitaria (Sunedu) presentó el Informe Bienal de la Realidad Universitaria, donde incluyó su ranking de las mejores universidades en el Perú a partir de una evaluación de Incites Bencharking \& Analytics, que a su vez recogió la data de publicaciones registradas por Web of Science Core Collection².

¿Cuáles fueron los indicadores medidos para realizar este ranking?. En primer lugar, el número de publicaciones relacionadas a cada universidad en revistas consideradas por la mencionada web y el número de documentos citables entre 2014 y 2016. Asimismo, el índice H de cada universidad entre 1996 y 2016, que es la suma de artículos científicos publicados y cuántas veces fueron citados, y el número de artículos publicados que se encuentran entre el 10\% de los más citados en Web of Science Core Collection, también entre 2014 y 2016. En dicha evaluacion la Universidad Ricardo Palma en Lima, Perú ocupaba el lugar 18.

En octubre, la revista América Economía compartió su propio ranking de las mejores universidades del Perú en el 2018, evaluando indicadores como calidad de los docentes universitarios, investigaciones e infraestructura, ocupando el lugar 18 de mas de 90 universidades evaluadas.

La buena noticia para la Universidad Ricardo Palma es que una reciente evaluación: Avance de la publicación científica de las universidades peruanas en Scopus a diciembre de 2018, (ver tabla 2) nos ubica en el lugar 15, subiendo 3 escalones en el tercio superior de las universidades del Perú, con un indicador promisorio de

\footnotetext{
'Director General del INICIB, URP, Lima-Perú.

Especialista en Oncología Medica.

Maestría en Investigación Clínica.

'Doctorado en Medicina.

Rector de la Universidad Ricardo Palma, Lima-Perú

d Bachiller en Educación y Título de Abogado.

e Doctor en Educación.

Investigador y Escritor.
}

Citar como: Jhony A. De La Cruz -Vargas, Elio Rodríguez-Chávez. La investigación: Más allá del ranking de las universidades [Editorial]. Rev. Fac. Med. Hum. 2019;19(1):7-12. (Enero 2019). DOI 10.25176/RFMH.v19.n1.1786 


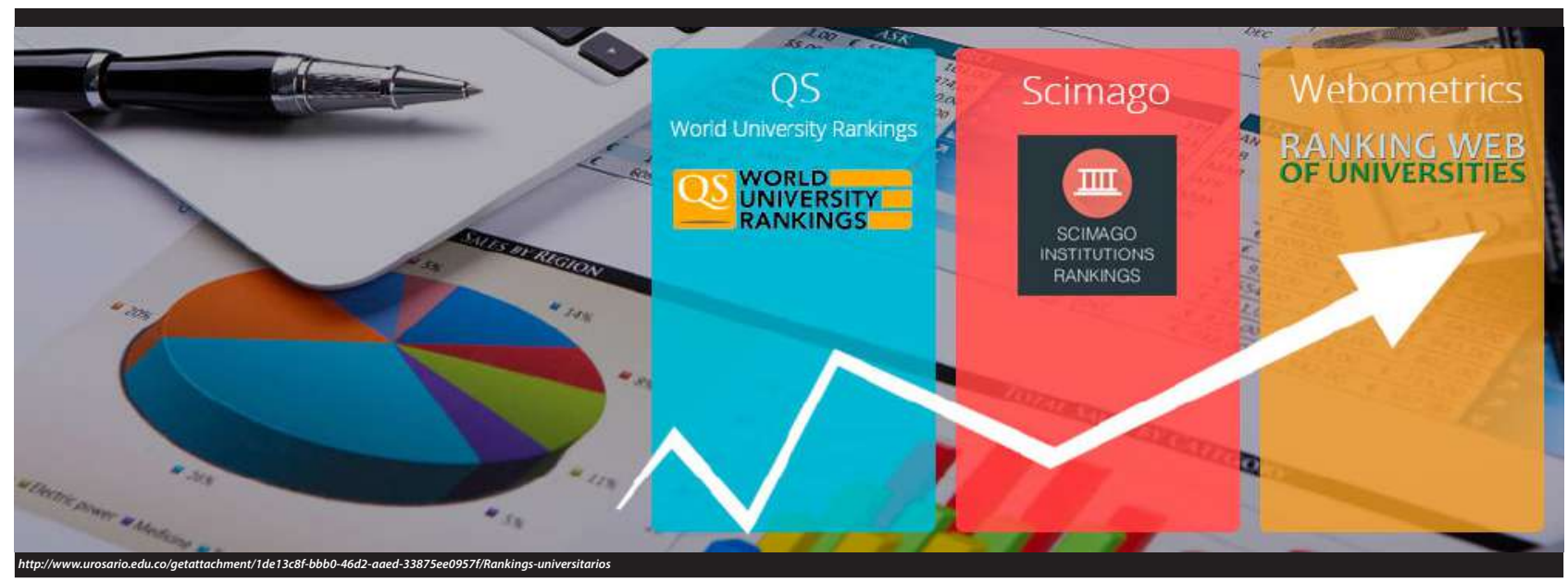

avance en publicaciones (mayor al 100\%) ${ }^{3}$. Siendo el área Biomedica una de las áreas con mayor poder de publicacion a nivel nacional e internacional.

\section{La investigación en las universidades privadas:} ¿Un lujo o una necesidad? ${ }^{5}$

La investigación, más álla del ranking, representa una de las necesidades más importantes para las universidades públicas y privadas. La relevancia que cada una de estas instituciones le otorga a este rubro, se ve claramente reflejada en la cantidad de artículos publicados, los miembros del Sistema Nacional de Investigadores (SINACYT) que tienen contemplados dentro de su plantilla académica, así como el número de patentes que les son otorgadas a partir del conocimiento obtenido. Todas las instituciones de educación superior deben contemplar en sus objetivos prioritarios, mejorar año tras año la infraestructura y el apoyo financiero a la investigación. Lo anterior resulta esencial para convertirse en universidades líderes, productoras de nuevo conocimiento y partícipes del desarrollo de su país, logrando reconocimiento como institución comprometida con la investigación científica y tecnológica.

La investigación está presente en la vida del hombre desde sus orígenes. Al investigar, brindamos a los demás un nuevo conocimiento sobre la realidad que nos rodea, pero también beneficiamos a la población $y$, sobre todo, le ofrecemos a nuestro país los elementos necesarios para progresar. Esto da lugar a lo que se conoce como "economía basada en el conocimiento", actividad que es ejercida por países como Estados Unidos (EE.UU) que desde siempre han mostrado un desarrollo tecnológico y competitividad muy elevados. EE.UU invierte más del $2.5 \%$ de su producto interno bruto (PIB) en actividades científicas ${ }^{6}$. En México, en el 2011 se invirtió solamente el $0.46 \%$ del PIB en ciencia, tecnología e innovación. En Europa, Finlandia y Suecia destinan más del 3.5\% a este rubro. El Banco Mundial afirma que el Perú invierte sólo $0.15 \%$ de su PBI en investigación y desarrollo, a pesar de que casi un tercio de la población son jóvenes buscando oportunidades de crecer académicamente. En Sudamérica, Brasil invierte más del $1 \%$, es decir, alrededor de un $0.90 \%$ más de lo que destina Perú a la investigación. Por supuesto que esta inversión reditúa a estos países una mayor cantidad de productos científicos, un buen desarrollo tecnológico y, desde luego, una mejor competitividad. En la mayor parte de los países desarrollados, la actividad científica se lleva a cabo de forma muy importante en las instituciones de estudios superiores (IES), especialmente en universidades privadas. Harvard, Cambridge, Princeton, la Universidad de Columbia y Yale son tan sólo un ejemplo del nivel de calidad que la investigación puede alcanzar en estos recintos de estudio. Estas universidades no sólo comparten el privilegio de estar clasificadas como instituciones con un gran nivel de investigación sino que, además, comparten el hecho de pertenecer al sector privado (U.S. News and World Report, 2012). En Estados Unidos un porcentaje muy alto de las publicaciones mensuales

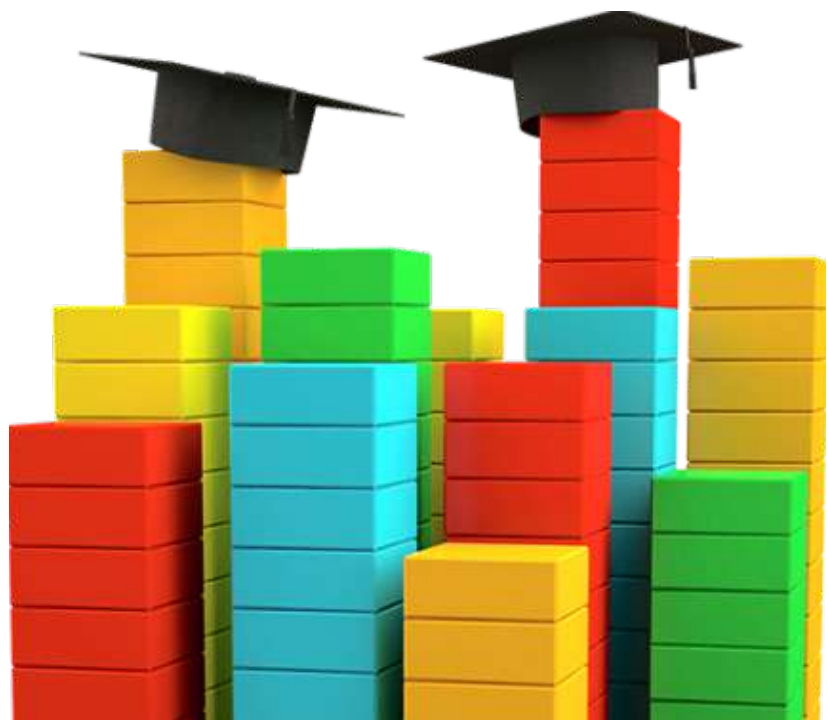


proviene de este tipo de universidades. Esto es, sin lugar a dudas, el resultado de la promoción e inversión que estas instituciones le conceden a la investigación.

En relación con la promoción, uno de los puntos fundamentales que estas universidades tratan de resaltar en sus instrumentos promocionales, es la actividad científica que desarrollan. La promoción de la actividad científica, no sólo la manejan para los cursos regulares, también manejan cursos especiales de verano a los que incluso invitan alumnos de pregrado a nivel mundial para convivir con científicos profesionales y así dar inicio a su desarrollo científico en laboratorios de alto nivel. En este sentido el Instituto de Investigacion en Ciencias Biomédicas (INICIB) de la Universidad Ricardo Palma, ha sido pionera en desarrollar "Cursos de Investigación y titulación por tesis y programas de investigación de verano", como las pasantias de investigación promovidas por Asociación peruana de facultades de medicina (ASPEFAM), donde estudiantes de medicina de diversas universidades del país completan una experiencia de investigación intensiva, ademas del desarrollo de una red de colaboración nacional e internacional.

En lo que respecta a la inversión, estas universidades consideran un fondo que deben invertir en lo que denominan prioridades institucionales. Como parte de estas prioridades, destaca el apoyo a propuestas de investigación y la compra de infraestructura científica, lo que representa para la universidad, una forma de mejorar su competitividad7. Esta claro que estas instituciones consideran a la investigación como algo más que un simple gasto, la ven como una necesidad urgente en las universidades privadas: Sólo por ejemplificar, la inclusión de programas científicos de alta calidad incrementa de manera importante el ingreso de alumnos de excelencia académica. Por otra parte, la inversión en líneas de investigación de alta calidad les redituará en la subvención de sus programas de posgrado y en la obtención a mediano y largo plazo de financiamientos extra institucionales y patentes que, sin ser muy exactos, recuperan a la institución el doble o incluso más de la cantidad invertida. Las universidades en Estados Unidos tienen muy claro que, invertir en actividades científicas representa una gran oportunidad de crecimiento. Es evidente que el producto de esta inversión se ve reflejado en el posicionamiento de estas universidades en el ranking mundial.

\section{La perspectiva de la investigación}

Resulta interesante obervar el modelo fundamentado en la vinculación con el ámbito empresarial, ésta es la forma en que muchas universidades han logrado colocarse a la cabeza de las universidades privadas en lo referente a la generación de productos científicos. No es casualidad que en Perú, a partir del 2017 Concejo Nacional de Ciencia, Tecnología e Innovación Tecnológica (CONCYTEC) y el Banco Mundial, lanzaron convocatorias dirigidas a fomentar proyectos de investigacion con la formula: Academia-Empresa.

En Latinoamérica se estima que, solamente una de cada diez investigaciones es desarrollada por instituciones privadas. Lo anterior deja clara la necesidad de impulsar la actividad científica en el sector privado.

En Perú, la cultura de las patentes está muy poco desarrollada, en la mayoría de los casos, el investigador piensa primero en publicar y muy pocas veces en patentar. Lo anterior se ve claramente reflejado en el incremento gradual de publicaciones a nivel nacional pero no así de patentes solicitadas por nuestros investigadores. Las patentes son indicadores de la investigación y el desarrollo tecnológico de un país. Es claro que estamos en un círculo vicioso en el que a menor presupuesto, menor investigación, menor conocimiento y por lo tanto menor número de patentes.

El número de investigadores peruanos por Universidad, en comparación con otros países, es muy reducido; sin embargo, año tras año se ha venido incrementando. A pesar de este incremento el número es muy reducido ya que representa alrededor del $0.01 \%$ del total de la población. Es evidente que nos encontramos ante la necesidad de desarrollar programas de estudio y actividades deinvestigación encaminadosalaformación de recursos La investigación en las universidades privadas: requiere de una estrecha colaboración entre los profesores adscritos, investigadores y estudiantes de pregrado y posgrado. Esto ya se ha comenzado en las universidades públicas y en algunas de las universidades privadas más reconocidas del país.

En las universidades privadas es mucho menor el número de investigadores que pertenecen al SINACYT. Uno de los principales problemas que origina que las universidades privadas cuenten con un menor número de investigadores registrados en CONCYTEC- SINACYT es la falta de recursos dirigidos a apoyar los proyectos de investigación en estas instituciones.

Es fundamental generar la importancia que debe recibir la visión del binomio universidad-empresa, un punto que probablemente sea muy poco valorado y sobre todo poco ejercido por muchas universidades privadas. Lo anterior podría explicar de alguna manera, los números reflejados en la cantidad de 
publicaciones y patentes, lo cual se ve fortalecido al contar con el apoyo económico del sector empresarial. Desafortunadamente en Perú, el apoyo económico del sector empresarial es muy bajo (<2.5\%), en comparación con otros países (mayor al 60\%), aún así, éste representa un gran apoyo para el desarrollo de protocolos científicos en aquellas universidades que recurren a empresas que apoyan este rubro y que están dispuestas a aportar el financiamiento necesario para llevar a cabo los proyectos de investigación. La empresa puede (y debe) acercarse al mundo académico para intentar resolver sus problemas, esos que su cotidianeidad y sus procesos les presentan, pero las políticas de investigación universitaria no pueden orientarse solo a resolver aquellos asuntos puntuales. La universidad puede buscar financiamiento empresarial para algunos ejes, pero, sobre todo, debe también financiar con sus propios recursos una investigación más teórica o aplicada, ejercida por sus institutos, docentes y alumnos.

\section{Necesidades y Reformas Necesarias:}

En Perú, y en las universidad privadas, las reformas deberán estar dirigidas a desarrollar el modelo de la triple hélice, un modelo donde las universidades, el sector empresarial y el gobierno interactúen de manera organizada para promover de manera integral, el avance cientifico y tecnológico.

Sin embargo, uno de las factores más importantes que influye en una institución para obtener patentes, miembros del SINACYT y artículos publicados es la infraestructura. Éste es uno de los puntos más importantes, ya que es posible que la institución cuente con proyectos bien estructurados y fundamentados, pero si no se cuenta con un laboratorio bien equipado o con los elementos necesarios para llevarlo a cabo, no se obtendrán buenos resultados y por lo tanto no será una investigación con la calidad requerida para ser publicada.

La investigación debería ser un tema de interés para nuestros jóvenes, ya que los alumnos son inventores potenciales, capaces de asegurar a las universidades como fuentes naturales de innovación. De esta forma, el campo de la investigación debería ser visto como uno de los pilares básicos de la educación y no como un requisito institucional necesario para cualquier tipo de acreditación de las carreras que ofrece. Es por esto que es muy importante que se introduzcan en las universidades peruanas asignaturas que impulsen la formación de nuevos investigadores, que se enseñe a los alumnos la importancia de la investigación, logrando que la vean no sólo como una materia que se debe cursar para seguir avanzando en la licenciatura, sino como una aportación de parte de ellos al avance científico. Sin duda alguna, más que un lujo la investigación debe ser considerada una necesidad real para las universidades públicas y privadas, especialmente para las privadas, ya que por el momento no siempre la han abordado de la manera más conveniente. Las universidades privadas en Perú y en casi toda Latinoamérica deben visualizar que la investigación es un rubro que puede lograr posicionarlas como instituciones líderes en el desarrollo de protocolos innovadores que, además de brindar nuevo conocimiento, sirvan como base para las investigaciones que se realizan en otros países. Debe hacerse investigación de alto nivel, que cuente con el financiamiento necesario para poder transformar ese conocimiento en tecnología aplicable a las necesidades que tenemos como país.

Desde las universidades debemos exigir en nuestros estudiantes y docentes esa capacidad de interpelación, de pensamiento crítico, así como promover el máximo nivel de calidad en las investigaciones para que puedan aparecer en revistas indizadas. En tanto más académicos peruanos estén presentes en ese tipo de publicaciones, nuestro sistema universitario tendrá mayor presencia en los rankings internacionales.

Pero, el ranking de las universidades, es solo el instrumento, y no el fin. El fin último es entender la investigación como parte esencial de la vida universitaria. El sistema debe organizarse con ese fin y no con el de aparecer en rankings. Lo segundo debe ser una consecuencia de lo primero; trabajemos asumiendo que, si la queremos sólida, no será inmediata. La lógica de los indicadores, muchas veces tan ilógica, no siempre refleja cuán fieles somos a la esencia de una institución. Está muy bien medir, pero está mejor saber qué queremos medir y para qué.

Finalmente, las IES, tanto públicas, como privadas deben convertirse en "universidades emprendedoras", que apuesten por la innovación, apoyen las nuevas propuestas y tengan la iniciativa para desarrollar nuevos protocolos y líneas de investigación para realizar investigación propiamente dicha y además se enseñe a los alumnos a cuestionarse y a preguntarse el porqué de la investigación en las universidades privadas. Sin embargo, muchas instituciones peruanas han pasado de noche las transiciones hacia la universidad de investigación, y de ahí hacia la universidad emprendedora. Muchas universidades por omisión, por inercia y por mandato, siguen siendo solo de docencia ${ }^{8}$. 
Deben enseñar a los alumnos a romper paradigmas y a proponer cosas nuevas, que le permitan al Perú dejar de ser un país consumidor de conocimiento y pasar a ser un país creador de su propia tecnología y portador de la misma a nivel mundial. Se debe considerar a la investigación como una de las armas más importantes que tiene el país para impulsar las ideas que miles de jóvenes tienen en mente y que podrían generar un cambio relevante en la forma en que se ve al Perú a nivel mundial. Se debe dejar atrás la idea de que la investigación es un lujo que genera gastos, para empezar a verla como una necesidad que además de posicionar a las IES como líderes en la creación de nuevo conocimiento, las puede hacer partícipes en el desarrollo de un Perú al que se le reconozca por su compromiso con la investigación científica y tecnológica para beneficio de la sociedad.

Tabla 1. Perú: Ranking de universidades - General.

\begin{tabular}{|c|c|c|c|c|c|c|c|}
\hline RANK & UNIVERSIDAD & $\begin{array}{c}N^{\circ} \mathrm{DE} \\
\text { AÑNOS AL } \\
2016\end{array}$ & $\begin{array}{c}\text { SEDE } \\
\text { (DEPARTAMENTO) }\end{array}$ & $\begin{array}{l}\text { TIPO DE } \\
\text { GESTIÓN }\end{array}$ & $\begin{array}{c}N^{\circ} \\
\text { PROGRAMAS } \\
\text { DE ESTUDIO } \\
\text { DE PREGADO } \\
\text { AL } 2015\end{array}$ & $\begin{array}{c}\text { TIPO DE } \\
\text { AUTORIZACIÓN / } \\
\text { LICENCIA }\end{array}$ & PUNTAJE \\
\hline 1 & Pontificia Universidad Católica del Perú & 99 & Lima & Asociativa & 63 & Licenciada & 100.000 \\
\hline 2 & Universidad Peruana Cayetano Heredia & 55 & Lima & Asociativa & 24 & Licenciada & 69.187 \\
\hline 3 & Universidad Nacional Mayor de San Marcos & 465 & Lima & Pública & 65 & Autorización Definitiva & 54.548 \\
\hline 4 & Universidad Nacional Agradia La Molina & 56 & Lima & Pública & 12 & Licenciada & 38.432 \\
\hline 5 & Universidad Nacional de ingenieria & 61 & Lima & Pública & 28 & Autorización Definitiva & 26.086 \\
\hline 6 & $\begin{array}{l}\text { Universidad Nacional de San Antonio Abad del } \\
\text { Cusco }\end{array}$ & 324 & Cusco & Pública & 40 & Autorización Definitiva & 23.584 \\
\hline 7 & Universidad Nacional de Trujillo & 192 & La Libertad & Pública & 45 & Autorización Definitiva & 20.045 \\
\hline 8 & Universidad Cientifica del Sur & 18 & Lima & Sociativa & 21 & Autorización Definitiva & 19.865 \\
\hline 9 & Universidad de Piura & 48 & Piura & Asociativa & 28 & Licenciada & 14.843 \\
\hline 10 & Universidad del Pacifico & 54 & Lima & Asociativa & 9 & Licenciada & 11.088 \\
\hline 11 & Universidad Nacional del Altiplano & 55 & Puno & Pública & 35 & Autorización Definitiva & 10.987 \\
\hline 12 & Universidad Peruana de Ciencias Aplicadas & 22 & Lima & Sociativa & 52 & Licenciada & 9.976 \\
\hline 13 & Universidad Nacional de la Amazonia Peruana & 55 & Loreto & Pública & 30 & Autorización Definitiva & 9.671 \\
\hline 14 & Universidad de San Martin de Porres & 54 & Lima & Asociativa & 23 & Licenciada & 8.183 \\
\hline 15 & Universidad de Lima & 54 & Lima & Asociativa & 11 & Licenciada & 7.585 \\
\hline 16 & Universidad Nacional de San Agustin & 188 & Arequipa & Pública & 47 & Autorización Definitiva & 7.562 \\
\hline 17 & Universidad Esan & 13 & Lima & Asociativa & 10 & Autorización Definitiva & 6.844 \\
\hline 18 & Universidad Ricardo Palma & 47 & Lima & Asociativa & 23 & Licenciada & 6.227 \\
\hline 19 & Universidad Católica San Pablo & 19 & Arequipa & Asociativa & 11 & Licenciada & 4.198 \\
\hline 20 & Universidad Privada San Ignacio de Loyola & 21 & Lima & Sociativa & 41 & Licenciada & 4.005 \\
\hline 21 & Universidad Nacional Federico Villareal & 53 & Lima & Pública & 67 & Autorización Definitiva & 3.909 \\
\hline 22 & Universidad Nacional de Piura & 55 & Piura & Pública & 35 & Autorización Definitiva & 3.097 \\
\hline 23 & Universidad Católica de Santa María & 55 & Arequipa & Asociativa & 28 & Autorización Definitiva & 2.765 \\
\hline 24 & Universidad Nacional Pedro Ruiz Gallo & 46 & Lambayeque & Pública & ND & Autorización Definitiva & 2.309 \\
\hline 25 & Universidad Privada Antenor Orrego & 28 & La Libertad & Asociativa & 23 & Autorización Definitiva & 1.565 \\
\hline 26 & Universidad Nacional del Callao & 50 & Lima-Callao & Pública & 17 & Autorización Definitiva & 1.235 \\
\hline 27 & Universidad Alas Peruanas & 20 & Lima & Sociativa & 31 & Autorización Definitiva & 0.819 \\
\hline 28 & Universidad Nacional de Tumbes & 32 & Tumbes & Pública & 20 & Autorización Definitiva & 0.439 \\
\hline 29 & Universidad La Salle & 5 & Arequipa & Asociativa & 3 & $\begin{array}{l}\text { Autorización } \\
\text { Provisional }\end{array}$ & 0.420 \\
\hline 30 & Universidad Privada San Juan Bautista S.A.C & 19 & Lima & Sociativa & 16 & Autorización Definitiva & 0.292 \\
\hline 31 & Universidad Andina del Cusco & 32 & Cusco & Asociativa & 18 & Autorización Definitiva & 0.281 \\
\hline 32 & Universidad Privada del Norte & 22 & La Libertad & Sociativa & 44 & Licenciada & 0.063 \\
\hline
\end{tabular}

Nota 1: Reporte generados sobre la base de los documentos indexados en la Web of Science Core Collection el 8 de setiembre del 2017.

Nota 2: Para tipo de Autorización / Licencia, la fecha de corte es el 11 de Noviembre del 2017.

Fuente: Inotes Benchmarking G. Analytics - Web of Sdence Core Collection SUNEDU.

Elaboración SUNEDU. 
Tabla 2. Avance de la producción científica de universidades peruanas en Scopus, diciembre 2018.

\begin{tabular}{|c|c|c|c|c|c|c|}
\hline & \multirow[b]{2}{*}{ UNIVERSIDADES } & \multicolumn{2}{|c|}{ TOTAL DE ARTÍCULOS } & \multicolumn{3}{|c|}{ SCOPUS 2017} \\
\hline & & 2018* & AVANCE** & DIC 2018 & DIC 2017 & AVANCE*** \\
\hline 1 & U. Peruana Cayetano Heredia & 350 & $(87,1)$ & 402 & 330 & $(82,1)$ \\
\hline 2 & Pontificia U. Católica del Perú & 349 & $(84,7)$ & 412 & 307 & $(74,5)$ \\
\hline 3 & U. Nac. Mayor de San Marcos & 341 & $(91,9)$ & 371 & 244 & $(65,8)$ \\
\hline 4 & U. Peruana de Ciencias Aplicadas & 147 & $(105,8)$ & 139 & 108 & $(77,7)$ \\
\hline 5 & U. San Martin de Porres & 100 & $(96,2)$ & 104 & 73 & $(70,2)$ \\
\hline 6 & U. Cientifica del Sur & 82 & $(132,3)$ & 62 & 43 & $(69,4)$ \\
\hline 7 & U. Nac de Ingenieria & 77 & $(120,3)$ & 64 & 54 & $(84,4)$ \\
\hline 8 & U. Nac de San Agustín & 70 & $(92,1)$ & 76 & 42 & $(55,3)$ \\
\hline 9 & U. Nac Agraria La Molina & 63 & $(82,9)$ & 76 & 62 & $(81,6)$ \\
\hline 10 & U. Nac San Antonio Abad & 63 & $(112,5)$ & 56 & 33 & $(60,0)$ \\
\hline 11 & U. del Pacífico & 60 & $(127,7)$ & 47 & 42 & $(89,4)$ \\
\hline 12 & U. San ignacio de Loyola & 48 & $(106,7)$ & 45 & 30 & $(66,7)$ \\
\hline 13 & U. Continental & 39 & $(108,3)$ & 36 & 24 & $(70,6)$ \\
\hline 14 & U. Nac Trujillo & 38 & $(92,7)$ & 41 & 31 & $(75,6)$ \\
\hline 15 & U. Ricardo Palma & 35 & $(116,7)$ & 30 & 20 & $(69,0)$ \\
\hline 16 & U. Privada del Norte & 33 & $(84,6)$ & 39 & 31 & $(79,5)$ \\
\hline 17 & U. de Piura & 25 & $(55,6)$ & 45 & 27 & $(60,0)$ \\
\hline 18 & U. ESAN & 23 & $(79,3)$ & 29 & 27 & $(93,1)$ \\
\hline 19 & U. Privada Antenor Orrego & 22 & $(50,0)$ & 44 & 22 & $(50,0)$ \\
\hline 20 & U. Nac Federico Villarreal & 21 & $(77,8)$ & 27 & 20 & $(74,1)$ \\
\hline
\end{tabular}

* Scopus 5 de diciembre 2018, a partir del ID affiliation de cada universidad

** Avance en relacion al año anterior (artículos 2018*100/artículos 2017)

***Avance de produccion del 2017 medido en diciembre 2017, sobre lo que finalmente tuvo, medido en diciembre de 2018

Conflicto de interés: elaborado por PMT, Director de Gestión de proyectos y promocion de la investigación de la Universidad Científica del Sur.

Correspondencia: Jhony A. De La Cruz Vargas

Dirección: INICIB, Facultad de Medicina Humana, Edificio 1-208. 2do piso. Avenida Benavides 5440, Surco, Lima-Perú.

Teléfono: 708-0000 / Anexo: 6016

Correo:jhony.delacruzv@urp.pe

\section{REFERENCIAS BIBLIOGRÁFICAS}

1. Rico, A.: 'Investigación en la universidad colombiana. Comunicación Educación, una relación estratégica', Revista Nómadas, 1996; Núm. 5, Bogotá.

Dispnible en: http://www.apostadigital.com/revistav3/hemeroteca/ jgpv1.pdf

2. Informe Bienal sobre la realidad universitaria

https://www.sunedu.gob.pe/informe-bienal-sobre-realidaduniversitaria/

3. Mayta Tristan: Avance de la publicacion cienitifica de las universidades peruanas en Scopus a diciembre de 2018.

Disponible en: https://twitter.com/maytatristan

4. Royero, J.: 'Gestión de sistemas de investigación universitaria en América latina'. Revista Iberoamericana de Educación, OEl, 2002: Núm. 30.

5. Ibarra Arias, José Juan Antonio, Villagrán Guerrero, Alejandra Elizabeth, La investigación en las universidades privadas: ¿un lujo o una necesidad?.
Universidades [en linea] 2013, (Enero-Marzo) : [Fecha de consulta: 12 de enero de 2019]

Disponible en:<http://www.redalyc.org/articulo.oa?id=37331244010>

6. El estado de la ciencia. Red Iberoamericana de Indicadores de Ciencia y Tecnologia. 2010.

Disponible en: http://www.ricyt.org/publicaciones/211-el-estado-de-laciencia-2010

7. Capaldi . "The Top American Research Universities", en The Center for Measuring University Performance, 2009. Arizona State University. http://mup.asu.edu/research2009.pdf

8. Arechavala Vargas, Ricardo. Las universidades y el desarrollo de la investigación científica y tecnológica en México: Una agenda de investigación. Rev. educ. sup, México , v. 40, n. 158, p. 41-57, jun. 2011. Disponible en: http://www.scielo.org.mx/scielo.php?script=sci_ arttext\&pid=S0185-27602011000200003\&lng=es\&nrm=iso 